Du code du barrio à l'idéologie d'une entreprise : l'extorsion et l'économie morale de la violence des gangs au Guatemala

From the Code of the Barrio to the Ideology of a Business: Extortion and the Moral Economy of Gang Violence in Guatemala City

\title{
Katherine Saunders-Hastings
}

Traducteur : Tom Cuthbertson

\section{(2) OpenEdition \\ Journals}

\section{Édition électronique}

URL : https://journals.openedition.org/conflits/20325

DOI : $10.4000 /$ conflits. 20325

ISSN : $1777-5345$

Éditeur :

CECLS - Centre d'études sur les conflits - Liberté et sécurité, L'Harmattan

\section{Édition imprimée}

Date de publication : 10 octobre 2018

Pagination : 121-140

ISBN : 978-2-343-16812-8

ISSN : 1157-996X

\section{Référence électronique}

Katherine Saunders-Hastings, « Du code du barrio à l'idéologie d'une entreprise : l'extorsion et l'économie morale de la violence des gangs au Guatemala », Cultures \& Conflits [En ligne], 110-111 | été/automne 2018, mis en ligne le 10 octobre 2020, consulté le 06 janvier 2022. URL : http:// journals.openedition.org/conflits/20325; DOI : https://doi.org/10.4000/conflits.20325 


\section{Du code du barrio}

à l'idéologie d'une entreprise : I'extorsion et l'économie morale de la violence des gangs au Guatemala 1

\section{Katherine SAUNDERS-HASTINGS}

Katherine Saunders-Hastings est enseignant-chercheur en études latino-américaines à l'Institut des Amériques de l'University College London (UCL), où elle enseigne l'anthropologie politique et urbaine. Ses recherches portent sur la violence urbaine et l'économie criminelle en Amérique latine, avec un intérêt tout particulier pour les quartiers de Guatemala-ville affectés par les gangs.

$\ll \coprod \begin{gathered}\text { e code du barrio a été troqué contre l’idéologie d'une entreprise ». } \\ \text { Ainsi Cadejo, ancien membre du Barrio 18, a-t-il décrit la trajectoire }\end{gathered}$ des gangs du Guatemala depuis les années 1990. À l'instar de beaucoup d'autres anciens membres que j'ai interviewés, Cadejo regrette ce qu'il perçoit comme l'abandon par son gang de normes qui naguère imposaient certaines limites à l'usage de la violence et aux cibles autorisées : un changement qu'il impute à l'émergence de l'extorsion comme activité principale du groupe.

Dans le « Triangle du Nord » centraméricain composé par le Salvador, le Honduras et le Guatemala, les gangs de rue dits maras (la Mara Salvatrucha abrégé en MS-13 et Barrio 18) ont énormément changé depuis leur apparition dans cette région, il y a environ vingt-cinq ans. Vu l'envergure de leurs économies criminelles, leurs répertoires d'actes violents et l'impact qu'ils ont sur les communautés urbaines pauvres, il est clair que « les gangs centraméricains d'aujourd'hui ne sont plus les gangs de rue légèrement délinquants d'il y a une génération ${ }^{2}$ ».

1. Texte traduit de l'anglais par Tom Cuthbertson.

2. Brenneman R., Homies and Hermanos: God and Gangs in Central America, Oxford, Oxford University Press, 2011, p. 14. 
S'appuyant sur une étude de terrain ethnographique de dix-neuf mois, menée entre 2011 et 2017 dans le quartier de Colonia el Romero à Guatemalaville ${ }^{3}$, cet article met en avant un aspect de cette transformation : l'évolution des rapports entre les cliques ${ }^{4}$ de la mara et leur barrio. En particulier, il suit le mouvement vers une économie fondée sur l'extorsion, qui a conduit des groupes autrefois fortement identifiés à leurs barrios à se diriger vers l'exploitation violente de ces mêmes quartiers. Ce virage prédateur infléchit l'expérience de l'insécurité dans les communautés marginalisées où se sont implantées les maras: les « zones rouges 5 » qui représentent l'épicentre statistique et discursif de l'insécurité urbaine.

Les habitants d'El Romero racontent souvent que la mara est devenue beaucoup plus dangereuse pour eux au cours des dernières années. Ils font part de l'inquiétude et de la détresse qui, pour eux comme pour Cadejo, sont imputables à une déstabilisation des normes qui naguère dictaient le rapport entre les gangs et leur environnement. Ces habitants expriment souvent le sentiment d'assister à une violation des liens normatifs et des limites éthiques, évoquant le déclin des « códigos de barrio » [codes de quartier] qui autrefois régissaient les responsabilités des uns et des autres, tant à l'intérieur de la communauté et des cliques de gang, qu'entre les deux. Cette expression désigne un ensemble de normes codifiant un «imaginaire social » local : une vision normative du vivre-ensemble 6 .

Les discours qui entourent les codes du barrio laissent entrevoir une sorte d'histoire morale d'El Romero et de son rapport avec les gangs présents sur place. Une histoire que cet article considère du point de vue des habitants du quartier et des membres de la mara, en s'intéressant d'abord à l'évolution des gangs de rue d'El Romero ainsi qu'à l'expansion du racket. Puis, à la façon dont ces mêmes habitants décrivent la teneur et le déclin des codes du barrio, ces cadres normatifs qui « permettent de distinguer les gens qui vivent à l'intérieur d'un groupe de ceux venus de l'extérieur 7 ». À partir des concepts de banditisme social et d'économie morale, je postule que l'un des grands changements survenus à El Romero au fur et à mesure de l'évolution de la mara concerne justement cette définition d'intérieur et d'extérieur aux yeux des habitants et des gangs qui partagent ce même espace. La séparation sociale et éthique que j'identifie ici s'explique à la fois par une transnationalisation de la

3. El Romero ainsi que tous les autres noms sont des pseudonymes. Sauf indication contraire, les citations sont tirées d'entrevues et de conversations réalisées avec les habitants du quartier. Je choisis de ne pas inclure les dates des entretiens ni d'autres mentions comme mesure d'anonymisation.

4. Les deux grandes maras sont composées de factions locales des gangs qui s'appellent cliques [clikas] et qui opèrent au niveau des quartiers ou des zones urbaines.

5. «Zona roja» désigne des quartiers (en général pauvres et urbains) connus pour des taux de violence, d'activités liées aux gangs et de criminalité très élevés.

6. Taylor C., « Modern social imaginaries », Public Culture, vol. 14, n¹, 2002, p. 106.

7. Pirie F., The Anthropology of Law, Oxford, Oxford University Press, 2013, p. 158. 
culture de gang perceptible à partir des années 1990 et par le recours de l'État guatémaltèque à l'incarcération de masse comme stratégie de répression. Le répertoire des violences qui, sous l'influence de l'extorsion, s'élargit et s'intensifie, témoigne de la transformation des imaginaires sociaux et des économies morales du crime dans les zones rouges de Guatemala, autant qu'il en est le producteur.

\section{Vivre dans les zones rouges}

\section{El Romero et ses gangs}

Les habitants d'El Romero décrivent leur quartier tristement célèbre avec ironie, le présentant comme « la plus rouge des zones rouges ». Depuis longtemps ancré dans les représentations populaires à Guatemala-ville en tant que berceau de la pauvreté, de la criminalité et de la violence, le barrio est situé dans la plus violente des vingt-deux zones urbaines de la capitale ${ }^{8}$. Des occupations de terrain souvent informelles, ainsi qu'une très forte mobilité résidentielle, font que les recensements de la population à El Romero soient peu fiables et deviennent rapidement obsolètes : les estimations varient entre 14000 et 34000 habitants.

Dans le passé, les garçons et les jeunes hommes d'El Romero s'organisaient le plus souvent en pandillas de cuadra (bandes de rue) à l'image des gangs que l'on trouve dans les quartiers populaires de Guatemala-ville depuis au moins les années 1950 9. Ses membres étaient recrutés dans des groupements sociaux très localisés ou parmi les adeptes de certaines sous-cultures ou activités adolescentes (break dance, heavy metal). Ces pandillas « se livraient à des infractions mineures et se bagarraient les unes avec les autres sans pour autant dominer [...] la vie du quartier et sans s'entretuer 10 ». Selon les habitants d'El Romero, en général ces pandillas s'abstenaient de s'en prendre aux riverains. Arturo, éducateur et organisateur local, se fait l'écho de cette impression quand il décrit les gangs qu'il connaissait durant son adolescence au cours des années 1980. Selon lui, leurs membres « insistaient vraiment sur la défense du barrio, pas seulement contre les autres cliques mais ils empêchaient aussi les vols, les agressions et les viols ».

8. Mendoza C.À. et Méndez C., « 7 mitos sobre la violencia homicida en Guatemala », El Periódico, 23 Janvier 2013; Dudley S., Homicides in Guatemala: The challenges and lessons of disaggregating gang-related and drug trafficking-related murders, InSight Crime, 2016, p. 28.

9. Pour désigner les gangs actuels, les termes pandilla et mara sont en général employés par les gens de façon interchangeable, tout comme les termes pandillero et marero pour désigner les membres des gangs.

10. Levenson D., Adios Niño: The Gangs of Guatemala City and the Politics of Death, Durham, Duke University Press, 2013, p. 32. 
S'il convient d'être prudent devant l'évocation de ce passé, sans doute un peu idéalisé, l'apparition au début des années 1990, en Amérique Centrale, de la MS 13 et de Barrio 18 a modifié la donne. À partir de cette période, ces maras ont rapidement essaimé en conjuguant une culture de gang importée de Californie du Sud avec des formes et des traditions qui existaient déjà dans ces régions. Les cliques des maras à Guatemala-ville ont pour la plupart été fondées par des adolescents guatémaltèques qui, sans quitter le pays, s'intégraient néanmoins à une communauté transnationale imaginée ${ }^{11}$. Cadejo évoque ainsi le retour à El Romero en 1993 d'un ami qui avait vécu à Los Angeles et qui racontait l'argot, les tatouages, les vêtements, les coupes de cheveux et la musique caractéristiques des maras «en los Estados Unidos». Telle était « la formule magique » pour les enfants et les adolescents du barrio et cette mode s'est ensuite imposée dans le quartier. Sur le fond, les cliques qui se sont multipliées à El Romero restaient des pandillas de cuadra, mais arboraient dès lors des signes d'appartenance à la MS 13 ou à Barrio 18 ainsi que des signatures stylistiques et des références culturelles empruntées. Selon Arturo, au début les gens voyaient ces maras comme « un truc plutôt marrant et inoffensif : elles voulaient protéger le barrio ». Un autre habitant de longue date rappelle que dans les années 1990 : « des maras il y en avait, mais des maras qui ne créaient pas d'ennuis».

Toutefois, au début des années 2000, l'exercice de la violence et les opérations criminelles des maras ont commencé à se renforcer. À partir de 2002, les cliques d'El Romero se sont affrontées au cours d'un conflit que les riverains ont appelé la guerre des maras et qui n'a pris fin qu'en 2008 quand une clique du Barrio 18 a éliminé ses dernières rivales au terme d'une campagne meurtrière. Depuis, cette même clique contrôle le quartier, dirige une économie criminelle basée sur l'extorsion des ménages, des commerces, des marchés, des transports et des services locaux. Tout comme dans d'autres territoires de gang à Guatemala-ville, la pratique de l'extorsion à El Romero implique des "sous-ensembles importants de la population 12 ». Un réseau de collaborateurs participe ainsi à la récolte de renseignements, à la distribution de demandes d'extorsion et à la collecte des paiements. D'âges très divers, allant de jeunes enfants à de vieilles dames, ces collaborateurs sont en effet beaucoup plus nombreux que les mareros eux-mêmes ${ }^{13}$.

11. Cruz J.M., «Maras and the politics of violence in El Salvador », dans Hazen J. and Rodgers D. (dir.), Global Gangs, Minneapolis, University of Minnesota Press, 2014, p. 126 ; Levenson D., op. cit., p. 10.

12. Fontes À., "Extorted life: Protection rackets in Guatemala City », Public Culture, vol. 28, $\mathrm{n}^{\circ} 3,2016$, p. 601.

13. Des collaborateurs ont avancé des estimations du nombre des membres à l'intérieur d'une clique qui variaient entre 80 et 300. Les divergences peuvent apparaître selon que le chiffre donné inclut les pandilleros incarcérés ou non, et en fonction des « rangs » qui sont pris en compte (certains degrés de participation n'octroyant pas un statut de membre de plein droit). 
Au quotidien, les conversations des habitants d'El Romero se portent sans cesse sur la question de l'évaluation et de la gestion des risques que représentent l'insécurité et les violences de gang. De même, quand les gens me parlent et quand ils discutent entre eux pour évoquer les changements qu'ils constatent dans le quartier, ils évoquent inlassablement la violence et les extorsions perpétrées par les gangs.

J'ai rencontré des hommes qui ont été membres de gangs locaux au cours des différents stades traversés par les cliques du quartier depuis les années 1980. Les entretiens qu'ils m'accordent ainsi que leurs propres discussions fournissent la matière permettant de restituer le point de vue des membres des gangs. Des anciens membres qui avaient participé aux cliques dans les années 1990 et au début des années 2000 (souvent rencontrés par l'intermédiaire de Cadejo ou de son ami Rafa, lui aussi ancien membre du Barrio 18) me parlent sans hésiter de leurs impressions des gangs actuels d'El Romero. Au fur et à mesure qu'ils apprennent à me connaître et à me faire confiance, ces mêmes hommes commencent à parler également de leur propre participation et des violences passées dont ils ont été auteurs ou victimes.

Il s'est par contre avéré plus difficile de rencontrer des membres actifs à des dates plus récentes. Au cours de la dernière décennie, l'attitude de la mara concernant le désengagement de ses membres s'est beaucoup durcie. En outre, il arrive souvent que ceux qui essaient de quitter la mara se cachent ou partent loin de chez eux afin d'éviter d'éventuelles représailles. J'ai néanmoins réussi à entrer en contact avec certains d'entre eux soit grâce à des membres de leurs familles ou des proches, soit grâce à des pasteurs évangéliques présents sur place qui m'ont permis de rencontrer quelques-uns de leurs paroissiens qui venaient de quitter la mara ou qui s'apprêtaient à le faire ${ }^{14}$. La plupart des témoignages sur les attitudes et les opérations de la mara au cours des dix dernières années proviennent de ces rencontres.

Les membres actifs se montrent en général hostiles envers les gens venus de l'extérieur. Le fait de divulguer des informations au sujet de la mara est passible de lourdes punitions. Cependant, en observant les comportements de mareros adolescents dans les rues d'El Romero j'en suis venue à mieux comprendre les relations qu'ils entretiennent avec les autres habitants du quartier. Certains collaborateurs impliqués dans la pratique de l'extorsion m'ont parlé ouvertement une fois que des proches ou des connaissances auxquels ils faisaient confiance avaient attesté de ma fiabilité. En outre, je me suis rendue dans plusieurs prisons proches de la capitale, ce qui m'a permis de me familiariser avec des espaces carcéraux où les maras dominent et ainsi de parler avec

14. À propos de la conversion évangélique et de la désistance, voir O’Neill K.L., Secure the Soul: Christian Piety and Gang Prevention in Guatemala, Berkeley, University of California Press, 2015 ; Brenneman R., op. cit. 
les membres en activité qui y sont incarcérés. Cependant, afin de minimiser les risques que cela représentait pour moi et pour les habitants du barrio perçus comme étant proches de moi, je n'ai pas cherché à établir de contacts ou à faire d'entretiens avec des membres de haut rang (qui en l'occurrence sont presque tous incarcérés). Ceci impose bien évidemment des limites à ma compréhension de la façon dont les actuels chefs ressentent et décrivent l'expansion du racket et la violence qui en découle ou leurs sentiments vis-à-vis des barrios où ils exercent leur pouvoir. Néanmoins, le large éventail de points de vue que j'ai pu recueillir au sein du barrio et de la mara me permet de bien décrire l'évolution du rapport qui existe entre les deux ainsi que la place que la violence et la prédation y occupent.

\section{Extorsion et insécurité dans un territoire mara}

Dans la mesure où elle permet de renforcer une mainmise sur les territoires urbains, de soutenir des pandilleros incarcérés et de garantir des salaires (quoiqu'en général en dessous du seuil de pauvreté) aux membres, l'extorsion s'est imposée au cours de la dernière décennie comme l'« élément vital de la criminalité » et le «moteur économique » des cliques des maras présentes dans le Triangle du Nord ${ }^{15}$. À l'époque de mon enquête de terrain, les tarifs journaliers variaient entre 10 et 15 quetzales (environ 1,5 dollars) pour les marchands ambulants et 30 quetzales pour les étals de marché, et des tarifs à partir de 50 quetzales s'appliquaient aux commerces, variant en fonction de la rentabilité de ces derniers aux yeux de la mara. Les transports représentent une source majeure de revenus : les chauffeurs de bus des lignes qui traversent El Romero payent environ $10 \%$ de leurs recettes alors que chacun des trente chauffeurs de moto-taxi travaillant dans le barrio paye un prix hebdomadaire de 500-800 quetzales ${ }^{16}$. La collecte de fonds auprès de familles considérées comme ayant des revenus réguliers peut avoir lieu chaque semaine, mais en général les ménages (tout comme les écoles et les associations présentes dans le quartier) sont obligés de verser une somme plus large de 5000 à 25000 quetzales (environ 600 à 3000 dollars) quand les gangs estiment - souvent selon des renseignements douteux - qu'ils disposent de biens, de fonds ou d'économies. L'expansion de l'extorsion et de la violence avec laquelle ce système est appliqué a aggravé l'impact des gangs sur la vie sociale et économique d'El Romero.

Selon les habitants d'El Romero, la sécurité et la qualité de vie dans le quartier ont diminué brusquement à mesure que l'extorsion s'est répandue

15. International Crisis Group (ICG), Mafia of the Poor: Gang Violence and Extortion in Central America, Latin America Report nº2, Bruxelles, 2017.

16. Les estimations des revenus liés à l'extorsion varient beaucoup. Certaines sources décrivent des tarifs plus élevés. Un chauffeur de bus a prétendu payer 37,5\% de ses recettes en « taxes de circulation": Levenson D., op. cit., p. 87. Les chiffres de la police indiquent des revenus tirés des lignes de bus inférieurs à ceux dont l'ont m’a fait part : Dudley S., op. cit., p. 29. 
sous l'égide d'une seule clique. Contrairement à la présentation faite des maras du passé comme étant des groupes qui « ne créaient pas d'ennuis », la délinquance d'aujourd'hui est, selon les dires d'un professeur local « d'une toute autre nature. Là, ils rackettent et tuent n'importe qui ». Colocho, trentenaire résident du barrio, m’a parlé de la même manière : pour lui, alors que la violence d'autrefois restait limitée et réglementée, "maintenant il n’y a plus aucun contrôle, c'est la loi de la jungle ».

Les habitants d'El Romero ont été très nombreux à fuir l'incertitude et l'insécurité qui règnent dans le quartier. Selon un recensement effectué par l'église catholique locale, la population du quartier aurait diminué de $20 \%$ entre 2008 et 2011. Dans certaines zones, un tiers des habitations ont été abandonnées. Beaucoup de ceux qui sont restés se montrent peu enclins à améliorer leurs conditions matérielles de façon trop visible par peur d'attirer l'attention des racketteurs. Comme le résume bien Arturo : "Si tu possèdes n’importe quel truc qui les intéresse - une voiture, une moto, si tu retapes ta maison - t'es foutu. Totalement foutu ». Beaucoup de commerces ont mis la clé sous la porte sous la pression des racketteurs et ceux qui restent sont aujourd'hui gérés par les familles de membres de gang. Les habitants se plaignent de la transformation de l'atmosphère du quartier, comparant les maisons abandonnées ou les rues désertes qu'ils voient autour d'eux et les fêtes de quartier, les rues débordant d'activité ou les portes toujours ouvertes d'autrefois. « À l'époque tout était vraiment joyeux - affirme une vieille dame avant de préciser -, je dis bien était parce que c'est fini maintenant».

Les habitants racontent que les pratiques violentes de la mara à leur encontre sont devenues beaucoup plus fréquentes et bien plus brutales que par le passé. Selon une jeune femme, "quand ils font quelque chose, c'est fait de façon vraiment atroce ». Le gang se sert de la violence de manière communicative et performative afin de faire respecter les extorsions, d'assurer une discipline interne et de démontrer aux habitants les conséquences éventuelles de la désobéissance. Pour rendre encore plus frappants ces usages de la violence, ils s'en prennent aux femmes et aux enfants. Lorsque le directeur d'une école locale n'a pas pu ou n'a pas voulu répondre à une demande d'extorsion, la cour de récréation a été mitraillée alors qu'elle était encore remplie d'enfants en pleine préparation de la semaine sainte. Une fois, un membre de la clique a fait une grave erreur et a été exécuté d'une balle dans la tête. Mais sa copine a été tuée de façon encore plus brutale : selon les dires d'un autre marero, son visage entier a été "effacé par les balles », histoire de bien montrer aux membres ce qui attendait leurs proches en cas d'erreur.

Selon les habitants d'El Romero, les meurtres perpétrés avec une violence extrême sont destinés à transmettre un message au quartier. Depuis le début des années 2000, le Guatemala s'alarme de l'apparition régulière de corps 
mutilés de jeunes femmes, souvent portant des signes d'agressions sexuelles ou de tortures ${ }^{17}$. Il existe des hypothèses et des théories du complot pour expliquer ces feminicides, mais à El Romero, où plusieurs corps ont été retrouvés, les explications sont claires : il s'agit de messages envoyés par la mara qui aime se servir de ces corps de jeunes femmes pour se faire comprendre de façon plus directe. Dans le sillage du meurtre d'une adolescente dont les parties du corps ont été retrouvées dans plusieurs lieux d'El Romero, les habitants m'ont dit qu'à leurs yeux, cela signifiait : «la mara est prête à tout, coûte que coûte, pour arriver à ses fins ».

\section{Les codes du barrio et l'éthique du crime}

\section{Les codes du gang et le virage vers la prédation}

Ce virage vers l'extorsion systématique de ces territoires a transformé la logique morale dominante de la violence de gang dans les zones rouges à Guatemala-ville. Les membres, actuels et anciens, que j'ai interviewés évoquent souvent les « codes du barrio » pour expliquer ces changements ${ }^{18}$. « Les codes sont différents », me dit-on. « Les codes ont changé ». «Il y avait d'autres codes ». Selon les dires de Murci, membre du Barrio 18 dans les années 1990, « la défense du barrio qui devait être protégé contre l'extérieur » était quelque chose d'obligatoire. Â la question de savoir s'il avait lui-même participé à la collecte de rentas (fonds extorqués), un contemporain de Murci, ancien membre d'une autre clique locale, a répondu : «Ben non, il fallait s'occuper du barrio ». Même si elle était sans doute respectée de façon toujours partielle, il existait néanmoins à cette époque une sorte de philosophie commune selon laquelle il était (pour citer les mots d'un autre ancien membre): «tout simplement de mauvais goût de piquer des choses aux gens qui t'entouraient, tu sais ?».

Les réponses données par des membres plus récents laissent en revanche entrevoir des changements profonds au niveau des comportements et des attitudes qui coïncident avec l'enracinement d'une économie fondée sur l'extorsion au cours de la dernière décennie. Les témoignages livrés par ces membres confirment une tendance identifiée par les spécialistes des gangs selon laquelle « les impératifs moraux » qui autrefois auraient structuré les cultures de gang

17. Voir, par exemple, Sanford V., «From genocide to femicide : Impunity and human rights in twenty-first century Guatemala », Journal of Human Rights, vol. 7, n², 2008, pp. 104-122; Constantino R., "Femicide, impunity, and citizenship : the old and new in the struggle for justice in Guatemala », Chicana/Latina Studies, vol. 6, n² 2, 2006, pp. 108-121.

18. Les spécialistes s'intéressent aux codes qui organisent les comportements des gangs depuis l'émergence des études de gang : voir, Thrasher F., The Gang, Chicago, University of Chicago Press, 1927. Les ethnographes se sont penchés également sur le rapport qui existe entre ces codes et un ordre social plus large et les mœurs en vigueur dans les quartiers occupés par les gangs : voir en particulier Anderson E., Code of the Street, New York, W.W. Norton, 1999. 
cèdent souvent la place à de nouveaux « impératifs économiques » à mesure que ces gangs s'orientent de plus en plus vers des fins criminelles ${ }^{19}$. Kevin, ayant adhéré à la clique du Barrio 18 d'El Romero à la fin des années 2000, a regretté la transformation de ces codes et l'abandon des normes régissant la vie de gang que ses frères aînés avaient connues par le passé. «Avant, le code nous disait de nous occuper du barrio et de ne pas emmerder les gens » a-t-il expliqué. Au lieu d'un système où les membres de gang faisaient respecter le barrio, il constate qu'aujourd'hui : « le plus souvent, tu le détruis ». Alors que les membres de la mara de la génération précédente prétendent en général n’avoir fait violence qu'aux gangs rivaux, Kevin admet avoir «fait beaucoup de mal à des gens qui n'avaient strictement rien à voir avec nos problèmes ». L'usage de la violence répond désormais à une logique d'extorsion. Comme l'explique Junior, membre adolescent : "Si tu tues quelqu'un ce n'est rien de personnel. C’est du business, c'était un ordre, ils n’ont pas payé. Ça fonctionne plus comme une entreprise ».

À une certaine époque, parmi les gangs à Guatemala, il y avait donc le sentiment qu'il était interdit de s'attaquer à son propre quartier et que celui-ci devait rester à l'abri des violences et de la criminalité. Ce sentiment commun a été bouleversé par l'expansion d'économies d'extorsion chez ces mêmes gangs. Dans un quartier contrôlé par la MS 13, les habitants ont cité des normes qui ressemblent à celles que l'on attribuait aux cliques d'El Romero autrefois. À cette époque, prétendait-on, les règles interdisant la prédation interne étaient respectées et appliquées scrupuleusement par des membres de gang qui représentaient l' « autorité » locale, une autorité qui passait non seulement par la force mais qui impliquait aussi une autorité morale. S'étant dirigé vers l'extorsion systématique de ces mêmes lieux, la clique a renoncé à ce rôle.

L'étude de longue haleine menée par Rodgers sur les gangs d'un barrio de Managua met en avant cette même tendance. Naguère, les pandillas du Nicaragua obéissaient à une "règle d'or [...]. Ne pas s'en prendre aux habitants de son propre quartier 20 ». Ici, comme ailleurs, la priorité donnée aux activités qui rapportent un profit au gang a transformé le rapport dominant de ce dernier avec le barrio. Poussé par la participation de ses membres dans l'économie de la drogue à partir de la fin des années 1990, le gang s'est mué d' " une organisation faisant preuve d'une certaine solidarité sociale avec la communauté locale en un groupe beaucoup plus exclusif et prédateur ${ }^{21}$ ».

19. Salagaev À.L. et Safin R.R., "Capitalizing on change : Gangs, ideology, and the transition to a liberal economy in the Russian Federation », dans Hazen J.M. et Rodgers D. (dir.), Global Gangs: Street Violence across the World, Minneapolis, University of Minnesota Press, 2014, p. 78.

20. Rodgers D., "Living in the shadow of death : Gangs, violence, and social order in urban Nicaragua, 1996-2002 », Journal of Latin American Studies, vol. 38, n², 2006, p. 275.

21. Rodgers D., "The moral economy of murder : Violence, death, and social order in Nicaragua ", dans Auyero J., Bourgois P., et Scheper-Hughes N. (dir.), Violence at the Urban Margins, Oxford, Oxford University Press, 2015, pp. 21-40, p. 31. 
Quand les habitants d'El Romero décrivent la brutalité dont ils sont l'objet, leurs mots s'accordent avec ce que l'on pourrait attendre d'un gang qui s'est transformé en « entrepreneur violent » se caractérisant par « la conversion d'une force organisée en revenu permanent $22 »$. Le rapport dominant entre la mara et le barrio est passé d'un modèle défensif à un modèle tributaire qui s'appuie sur la violence. Le changement qui s'est opéré aux yeux des gangs locaux dans la signification du concept de territoire a eu des conséquences considérables pour ceux qui vivent dans les zones de la ville qu'ils revendiquent.

\section{Les codes $d u$ barrio et l'imaginaire social}

Pour les habitants d'El Romero, l'abandon des codes du barrio a changé non seulement le rapport entre les gangs et le quartier mais aussi celui qui existe entre voisins. L'expression código de barrio désigne aussi bien les normes internes des gangs qu'une vision normative plus large du quartier. Elle s'emploie pour désigner à la fois un principe fondamental de sens moral et une éthique mettant l'accent sur la confiance sociale, l'entraide et la réciprocité locale. À en croire Cadejo, à l'époque de sa jeunesse dans les années 1980 et 1990, ces codes désignaient « une forme de solidarité entre tout le monde dans le quartier ». Mono, activiste adolescent, a donné une définition comparable : « autant de liens sociaux à l'intérieur de la communauté - ce sentiment de solidarité ».

Mais l'époque de cette solidarité généralisée serait largement révolue. Selon Mono : «maintenant, c'est sauve-qui-peut ». Les habitants d'El Romero imputent le démantèlement de cette philosophie communautaire aux extorsions, aux punitions et aux réseaux omniprésents de collaborateurs de la mara. Selon eux, chassés par la peur et la méfiance, les gens se sont retirés des espaces publics pour se renfermer, ce qui a ébranlé les réseaux sociaux indispensables qui autrefois avait permis un système d'entraide au quotidien. Ces affirmations coïncident avec ce que l'on constate dans d'autres régions d'Amérique centrale où la participation accrue des gangs aux économies criminelles a conduit à la transformation non seulement de ces groupes mais aussi plus globalement du «paysage moral » des communautés ${ }^{23}$.

Les códigos de barrio peuvent avoir plusieurs acceptions possibles. Quand les anciens ou actuels membres ont essayé de définir l'expression, ils ont évoqué pour la plupart les règles qui organisent et délimitent l'usage de la violence de la part des - ou entre les - gangs ainsi qu'une attitude protectrice envers leurs propres territoires. Quand d'autres gens ont expliqué le concept, ils ont

22. Volkov V., Violent Entrepreneurs: The Use of Force in the Making of Russian Capitalism, Ithaca, Cornell University Press, 2002, p. 28.

23. Rodgers D., "The moral economy of murder ", op. cit., p. 36 ; Hume M., The Politics of Violence, Oxford, Wiley-Blackwell, 2009, p. 107. 
cité en général des valeurs individuelles ou collectives plus larges : la communauté, l'autonomie, l'indépendance et l'entraide. Même si les avis sont divisés sur leur teneur exacte, il existe un consensus implicite sur le sens fondamental de ces séries de normes, responsabilités et croyances. Ces définitions s'accordent dans la mesure où elles tracent des frontières morales autour du quartier, le désignant en quelque sorte comme un domaine normatif, un élément à l'intérieur duquel la réciprocité est obligatoire et où la prédation est soit répréhensible soit inadmissible.

Même si la trajectoire générale esquissée par les présentations faites des códigos de barrio et de leur éventuel déclin reste plausible, il est désormais presque impossible d'établir avec certitude dans quelle mesure les « codes » du barrio, tels qu'ils existent aujourd'hui, correspondent à des normes qui dans le passé ont réellement réglementé les comportements et organisé la vie sociale dans le quartier ${ }^{24}$. Les avis restent partagés sur la question de la prédation. Selon Cadejo, peu importe l'époque, les gens se sont toujours fait voler à El Romero. Son ami Wuicho, qui a grandi dans le quartier au même moment, nuance : "avant on ne se faisait pas voler dans la colonia ». D'autres ont mis en question l'impact réel de la transformation de la mara sur les habitants du quartier. "Cela a changé - a reconnu une femme résidente dans le quartier depuis 33 ans - mais pas beaucoup. Il y a toujours eu des morts ».

L'analyse de ces déclarations contradictoires permet de distinguer deux visions du passé du quartier, l'une mettant l'accent sur les faits, établis, qui se sont produits dans ces lieux, l'autre s'intéressant plutôt au monde moral particulier qui y existe. Les incidents de prédation interne comme ceux cités par Cadejo ne remettent pas automatiquement en cause la vision générale des codes du barrio à laquelle faisait sans doute référence Wuicho. Les normes sociales coexistent depuis toujours avec des gens qui les enfreignent et qui refusent d'y voir des standards. De la même manière, suggérer que la violence a toujours existé ne veut pas dire pour autant que cette violence ait toujours pris la même forme ni eu la même signification. Même si le nombre de morts violents reste constant, ce que ces morts peuvent nous apprendre sur les normes autour de la violence et de la réciprocité peut très bien avoir totalement changé ${ }^{25}$. Quand les gens ont raconté que ces mêmes normes avaient été violées, ils ne prétendaient pas assister à l'émergence de comportements totalement nouveaux. En revanche, ils ont décrit l'affaiblissement d'un certain consensus sur le modèle de leur communauté et sur les rapports existant en son sein.

24. Sur ce point et sur la place occupée par la nostalgie dans les récits des codes de barrio, voir Saunders-Hastings K., " Red zone blues: Violence and nostalgia in Guatemala », Ethnography, OnlineFirst, 2018, https://doi.org/10.1177/1466138118795975.

25. Rodgers D., "The moral economy of murder ", op. cit. 
Le concept de banditisme social nous est utile pour comprendre la fracturation de l'imaginaire social et la transformation des moralités de la violence décrites par les habitants d'El Romero. Non pas que les maras, actuelles ou anciennes, doivent être considérées comme étant composées de bandits sociaux, mais parce que cette notion permet de considérer le rapport qui existe entre les gangs et les communautés d'où ils sont issus et où ils opèrent.

Comme le définit Hobsbawm, le bandit social est « un paysan hors-la-loi que le seigneur et l'État considèrent comme un criminel, mais qui demeure à l'intérieur de la société paysanne, laquelle voit en lui [...] un homme qu'il convient d'admirer, d'aider et de soutenir 26 ». Des critiques ont vu en lui « un pur produit de l'imagination humaine » qui ne ressemble à aucun hors-la-loi réel mais, de façon plus invraisemblable, à « un voleur noble, incarnant les valeurs d'un Robin des Bois 27 ». Néanmoins, ils tendent à ne relever qu'un seul aspect de la définition proposée par Hobsbawm : l'approbation et le soutien que de tels bandits auraient reçu de la part de la population locale. Je préfère insister en revanche sur l'idée d'un bandit qui demeure à l'intérieur de la société locale. En ne cherchant qu’à savoir si un hors-la-loi était «idolâtré » ou « criminalisé 28 » dans l'opinion populaire, nous risquons de passer à côté de comportements qui sont soit neutres d'un point de vue moral soit susceptibles d'être perçus comme des transgressions mineures plutôt que comme des péchés mortels. Néanmoins, du point de vue de ceux qui partout dans le monde partagent des territoires avec eux, c'est peut-être justement cette zone grise qui est occupée par de nombreux gangs de rue.

En ce qui concerne les bandits sociaux, la question cruciale ne devrait pas être de savoir s'ils sont admirés mais si, aux yeux des pauvres, ils continuent de faire partie de la communauté. Les gangs d'El Romero n'ont jamais bénéficié du même soutien populaire dont parle Hobsbawm. Cependant, jusqu'à son virage prédateur vers l'extorsion, la mara continuait à satisfaire aux critères généraux du banditisme social, dans la mesure où elle demeurait à l'intérieur du monde social et éthique du quartier. À l'image des bandits sociaux qui "partagent le système de valeurs des paysans ordinaires ", autrefois dans le Triangle du Nord les valeurs de la mara «se démarquaient peu de celles du barrio 29 ». À la fin des années 1980, les membres de gang à El Romero se rangeaient du côté des «pauvres méritants 30 ». Ils se considéraient et étaient

26. Hobsbawm E., Bandits, Londres, Abacus, 2001, p. 20.

27. Blok À., "The peasant and the brigand: Social banditry reconsidered », Comparative Studies in Society and History, vol. 14, ${ }^{\circ} 4,1972$, p. 500 ; Wagner K., "Thugee and social banditry reconsidered ", The Historical Journal, vol. 50, n², 2007, p. 353.

28. Wagner K., op cit., p. 370.

29. Hobsbawm E., op. cit., p. 43 ; Brenneman R., op. cit., p. 37.

30. Levenson D., op. cit., p. 67. 
considérés comme appartenant à la même sphère de réciprocité que tous les autres habitants du quartier.

Cela ne veut pas dire pour autant qu'un tel rapport soit sans antagonisme. Dans le passé, les membres de gang se comportaient toujours de manière à déplaire aux autres habitants du quartier, violant certaines de leurs valeurs et s'attirant leur désapprobation. Ils renversaient les attentes autour du passage des garçons et des jeunes hommes à l'âge adulte, à l'emploi et à la vie de famille. Il n'en demeure pas moins qu'ils partageaient une certaine vision de la solidarité de quartier et continuaient à satisfaire aux « conditions morales fondamentales de la réciprocité » au sein de cette communauté ${ }^{31}$.

En outre, le fait d'adhérer à un gang ne conduisait pas forcément à un changement radical de statut à l'intérieur du monde social du quartier. Il s'agissait d'un « rôle social défini », d'une identité qui n'était ni permanente ni exclusive ${ }^{32}$. Dans les années 1990, des membres de gang participaient également à des groupes ecclésiastiques, à des équipes sportives ou à des projets artistiques. Une fois passée la vingtaine, la plupart des membres renonçaient à participer pour retrouver leurs proches et pour réintégrer d'autres réseaux dans la communauté. Même si le fait d'avoir participé à ces gangs restait parfois mal perçu par les autres habitants du quartier, ce fait ne résumait pas à lui seul l'identité de ces membres et ne dictait pas non plus ce qu'ils allaient devenir.

Au départ donc, les maras appartiennent à ce contexte social plus large. Elles respectent les tabous qui les empêchent de trop s'en prendre aux autres à l'intérieur de ce même groupe, faisant preuve ainsi de « ces rapports d'un type particulier qui font qu'un bandit est social 33 ». Pourtant, de toute évidence, ces rapports ne tiennent plus à El Romero. Désormais, les gangs ressemblent davantage aux «voleurs professionnels et aux pillards » qui, dans la présentation d'Hobsbawm, tranchent avec les bandits sociaux. Ceux-ci sont définitivement à l'extérieur de la communauté des pauvres et forment leurs propres «anti-sociétés ». Ils se considèrent comme séparés des populations parmi lesquelles ils opèrent et auxquelles ils s'attaquent, qui les voient à leur tour comme des « criminels dans le sens qu'ils attribuent à ce terme et non pas seulement parce que la loi les reconnaît comme tels 34 ». Le banditisme social reconnaît une éventuelle "moralité différenciée de la violence » entre les élites et le peuple ${ }^{35}$. Mais pour avoir une vision complète de l'insécurité aux marges urbaines, il est au moins aussi important de reconnaître les différentes éthiques

31. Scott J.C., The Moral Economy of the Peasant, New Haven, Yale University Press, 1976, p. 42 .

32. Rodgers D., "Living in the shadow of death », op. cit., p. 284.

33. Hobsbawm E., op. cit., p. 20.

34. Hobsbawm E., op. cit., pp. 42-43, 20.

35. Rodgers D., « The moral economy of murder », op. cit., p. 30. 
de la violence et de la prédation qui distinguent les bandits sociaux des criminels souterrains. À la différence de ces derniers, les bandits sociaux ont en commun avec les pauvres une même morale de la violence.

Lorsque Cadejo explique que les maras ont échangé le « code du barrio » contre l'" idéologie d'une entreprise », ses propos doivent être compris à la lumière du démantèlement de l'économie morale dans les territoires des gangs à Guatemala-ville. Comme l'ont précisé E.P. Thompson et ensuite James Scott, le concept d'économie morale désigne un ensemble de critères visant les rapports entre les classes sociales autour de la production, la distribution et la commercialisation de la nourriture. Les pauvres se servaient de ces critères afin d'établir des limites entre « les pratiques légitimes et illégitimes » et entre ce qui était «tolérable et ce qui était intolérable » en termes de revendications sur leur production ${ }^{36}$. Ces économies étaient morales dans la mesure où ces normes, tout comme les códigos de barrio d'El Romero, s'articulaient autour d'un axe discursif central de réciprocité sociale instaurant les attentes, les obligations et les droits de chacun. Les relations économiques dans ces lieux étaient ainsi « ancrées dans des attentes morales sur les comportements appropriés qui obligeaient les puissants à rendre des comptes aux pauvres 37 ». Ayant "violé tous ces codes qui existaient ", c'est justement cette obligation de rendre des comptes au quartier dont la mara s'est défaite.

L'analyse historique de la foule anglaise proposée par Thompson et l'étude ethnographique de Scott sur les paysans vietnamiens portent sur les rapports qui existaient entre les classes sociales, sur l'empiétement d'économies capitalistes sur les relations traditionnelles en matière de production et de distribution de ressources, ainsi que sur l'émergence d'actions collectives ou de formes de résistance à de tels changements. Des travaux d'anthropologie plus récents ont élargi les fondements théoriques et le cadre analytique du concept d'économie morale au-delà des conflits entre les classes pour ensuite considérer la « régulation morale de la réciprocité à l'intérieur des classes sociales au quotidien 38 ». C’est dans ce sens qu'il est employé ici, suivant la définition proposée par Fassin : « la production, la circulation et l'utilisation des sentiments moraux, des émotions et des valeurs, des normes et des obligations dans l'espace social 39 ».

36. Thompson E.P., "The moral economy of the English crowd in the eighteenth century », Past and Present, vol. 50, 1971, p. 79; Scott J.C., op. cit., p. 3.

37. Karandinos G., Hart L., Montero F., and Bourgois P., « The moral economy of violence in the US inner city », dans Auyero J., Bourgois P., et Scheper-Hughes N. (dir.), Violence at the Urban Margins, Oxford, Oxford University Press, 2015, p. 43.

38. Karandinos G. et al., op cit., p. 45.

39. Fassin D., «Les économies morales revisitées », Annales Histoire, Sciences Sociales, vol. 64, n6, 2009, p. 1257. 
À la base de ce concept, il y a cette affirmation qu'une sphère d'activité qu'elle soit située à l'intérieur ou entre les groupements de classe - est « encastrée », étant «imprégnée par les normes en vigueur dans la société qui l'entoure 40 ». En ceci, l'économie morale se démarque de celle de marché, prétendument sans entraves et "désencastrée » ${ }^{41}$. Dans la première, les rapports d'échange s'inscrivent dans des «schémas de contrôle social et de réciprocité » plus larges et s'expriment en termes de «droits ou d'attentes moraux 42 ». De cette manière, rien n'empêche que les économies criminelles soient aussi des économies morales. Le bandit social qui ne dépasse pas les limites de la moralité en vigueur dans sa communauté, même s'il viole certaines normes légales, et le voleur professionnel ou le pillard, qui viole toutes les normes, correspondent de ce point de vue à une criminalité respectivement encastrée et désencastrée. Ce vocabulaire nous permet de comprendre les changements que les habitants ont décrit en évoquant l'abandon des codes du barrio.

Toutefois, ce passage d'une communauté morale à la séparation normative des maras de leur barrio, demeure trop schématique. Pour les habitants des communautés d'où ils sont issus, les membres de gang sont des «proches dangereux » (dangerous intimates), se caractérisant à la fois par la familiarité et l'altérité, la confusion et la contradiction ${ }^{43}$. Cela a d'ailleurs toujours été le cas à El Romero. Il n'empêche que, à en croire les récits que l'on entend sur la perte des codes et sur la montée de la violence, quelque chose semble avoir changé dans ce rapport, transformant ainsi cette constellation d'intimités et de dangers.

\section{« Désencastrer » la violence des gangs}

\section{Comment expliquer la dérive normative}

Cerner l'ensemble des dynamiques, des opportunités et des contraintes qui ont contribué à la transformation de la culture de groupe et de l'éthique criminelle de la mara dépasse le cadre de cet article ${ }^{44}$. Parmi les changements qui ont permis aux maras d'accroitre leur niveau de violence et les ressources dont elles disposent, et ce faisant d'atteindre le niveau de pouvoir et de contrôle qu'elles connaissent aujourd'hui, on peut mentionner la disponibilité accrue d'armes à feu à partir de la fin du conflit qui a dévasté le Guatemala entre 1960 et 1996 ainsi qu'une augmentation massive du flux de cocaïne tran-

40. Booth W.J., "On the idea of the moral economy ", American Political Science Review, vol. $88, n^{\circ} 3$, p. 664.

41. Polanyi K., La Grande transformation. Aux origines politiques et économiques de notre temps, Paris, Gallimard, 1983 [1944].

42. Scott J.C., op. cit., p. 40.

43. Penglase R.B., Living with Insecurity in a Brazilian Favela, New Brunswick, Rutgers University Press, 2014.

44. Voir Levenson D.T., op. cit.; Fontes A., Mortal Doubt: Transnational Gangs and Social Order in Guatemala City, Berkeley, University of California Press, 2018. 
sitant vers le nord à partir du début des années 2000. La décision prise par l'État guatémaltèque de privilégier l'incarcération à la place d'autres stratégies de répression a également joué un rôle décisif dans la séparation sociale et normative des cliques de la mara de leurs quartiers.

À partir du début des années 2000 quand les politiques de Mano dura ont fait grimper la population carcérale de façon exponentielle ${ }^{45}$, les prisons $\mathrm{du}$ Triangle du Nord sont devenues le berceau de la nouvelle implantation des maras dans la région. Bien que les chefs des deux maras soient actuellement presque tous incarcérés dans des prisons à sécurité maximale, les autorités du Guatemala ne sont pas parvenues pour autant à entraver la capacité opérationnelle de ces gangs. D'autant plus que, suite à l'introduction de prisons séparées pour les membres des deux maras à partir de 2005, le système pénitentiaire a regroupé des cliques éparpillées, leur permettant ainsi des contacts plus proches et durables, ce qui a favorisé la portée régionale, les bases de ressources et l'intégration opérationnelle de ces groupes. Mise en œuvre afin d'éviter une série d'affrontements meurtriers entre détenus, la répartition des membres rivaux dans des ailes et des établissements séparés a réduit la violence carcérale à court terme, tout en ayant pour effet pervers d' " augmenter la force des gangs à l'intérieur comme à l'extérieur des prisons 46 ».

La perméabilité des prisons du Guatemala n'entrave guère les réseaux de communication et de contrôle émanant de l'intérieur. Les chefs incarcérés dirigent les opérations menées dans la rue de très près, restant en contact constant avec les cliques. Comme l'explique Junior, membre du Barrio 18 âgé de dix-huit ans : " chaque ordre vient de la prison. Sans cet ordre, tu ne tires sur personne. Personne ne collecte rien sans en avoir reçu l'autorisation ». Plusieurs membres ou associés m'ont expliqué que sous l'influence d'une telle chaîne de commandement, imposée avec sévérité, les membres sur le terrain ne sont plus à même de mettre leur veto ou de contester l'application, l'expansion ou les cibles éventuelles de l'extorsion. Un membre subalterne a décrit les membres de son rang " comme des soldats : les ordres tu ne les mets pas en cause, tu les exécutes ».

45. L’année 2003-2004 a vu se produire plus de 22000 détentions, rien que dans le département de Guatemala, sous les auspices du Plan Escoba, qui ciblait les membres des maras. Le taux d'incarcération a continué à grimper depuis, avec une hausse de 150 \% entre 2008 et 2016. Cruz J. M., "Government responses and the dark side of gang suppression in Central America ", dans Bruneau T., Dammert L. et Sinner E. (eds.), Maras: Gang Violence and Security in Central America, Austin: University of Texas Press, 2011, p. 144; Prison Insider " Prisons in Guatemala », 2017, https://www.prison-insider.com/countryprofile/ prisonsinguatemala.

46. Lessing B., "The danger of dungeons: Prison gangs and incarcerated militant groups ", dans Krause K. (ed.), Small Arms Survey 2010: Gangs, Groups, and Guns, Cambridge, Cambridge University Press, 2010, p. 158. 
Des spécialistes des gangs du monde entier s'accordent à dire qu'au lieu d'affaiblir ces groupes, l'incarcération de chefs et de membres les renforcent ${ }^{47}$. Les études existantes se concentrent en grande partie sur des changements d'ordre pratique, pour souligner comment l'incarcération augmente la capacité des gangs en leur fournissant des «QG tactiques » ainsi que d'autres « outils opérationnels 48 ». Pourtant, la conséquence la plus significative du déplacement du centre de gravité des maras vers le système carcéral concerne probablement les cultures de groupe. Le gang s'est désancré de l'espace physique et social de son barrio.

Les origines de cette dérive remontent en effet au début des années 1990 et à l'émergence d'une culture de gang transnationale au sein des maras alors en pleine croissance. Malgré certaines continuités par rapport aux gangs de rue qui les ont précédés, l'émergence de Barrio 18 et de la MS 13 a eu pour conséquence une redéfinition des paramètres de l'identité, de l'appartenance et de l'hostilité aux yeux des membres. Auparavant, pour ces derniers le fait d'adhérer au gang et le fait d'habiter le quartier étaient largement synonymes, les deux réalités étant résumés par la phrase «mi barrio ». Barrio veut dire quartier qui veut dire gang : gang veut dire quartier qui veut dire barrio. L'importation d'une culture de gang californienne a pourtant introduit de nouvelles « normes, valeurs et connaissances en ce qui concerne les comportements ou bien la différence entre amis et ennemis 49 ». Pour ces groupes, le point nodal de l'identité et de l'appartenance s'est ainsi déplacé de plus en plus du quartier vers la mara.

Dorénavant, les violences perpétrées par un gang à l'encontre d'un autre gang servent moins à défendre un quartier qu'à affirmer ou à renforcer une identité franchisée. Une séparation s'est produite entre barrio au sens d'une appartenance à un quartier et barrio au sens d'une appartenance à un gang en particulier. Le changement qui s'est ainsi opéré dans le sens dominant de « $m i$ barrio » laisse entrevoir un déracinement de plus en plus prononcé des cliques de la géographie physique et sociale de leur quartier. Accéléré par un recours croissant à l'incarcération de masse de la part de l'État et à de nouvelles stratégies économiques de la part des gangs, ce déracinement a favorisé les violences prédatrices perpétrées à l'encontre de ces mêmes territoires au cours de la décennie suivante.

En raison de la concentration des structures organisationnelles et du noyau culturel de la mara dans les prisons, la plupart des décideurs « ne

47. Voir par exemple Lessing B., op. cit.; Hagedorn J.M., A World of Gangs: Armed Young Men and Gangsta Culture, Minneapolis, University of Minnesota Press, 2008, p. 13 ; SánchezJankowski M., "Gangs and social change », Theoretical Criminology, vol. 7, n², 2003, pp. 206-208 ; Salagaev À.L. et Safin R.R., op. cit., p. 72.

48. Lessing B., op. cit., p. 178.

49. Cruz J. M., « Maras and the politics of violence in El Salvador », op. cit., p. 126. 
s'identifient plus à ceux qui vivent dans les zones sous leur contrôle 50 ». À la fin des années 1980 et au début des années 1990, les membres s'identifiaient toujours à leur quartier : pour eux, «nous » désignait également les pauvres ${ }^{51}$. Mais, incarcérés dans des prisons séparées, les membres de gang voient leur modèle social réduit à l'échelle de la mara, ce qui renforce leurs liens avec les autres membres tout en fragilisant d'autres sentiments d'appartenance. C'est ainsi que le gang et le barrio ont connu une forme de « mitose normative 52 » : une rupture de l'imagination éthique auparavant partagée. Les gangs locaux ne souscrivent plus à cette vision du quartier comme une unité produisant des rapports et des devoirs moraux. Pour eux, le barrio ne fait plus partie du monde de réciprocités et de responsabilités qu'ils occupent.

\section{Violence et réciprocité}

Jusqu'ici, la violence a été décrite comme étant en grande partie un produit ou une conséquence de la séparation sociale et éthique entre les gangs et le barrio associée au virage vers la prédation des maras. Pour conclure, je chercherai à savoir dans quelle mesure la violence peut également être considérée comme productrice de cette même séparation.

Les études menées par Simon Harrison sur la guerre en Mélanésie peuvent nous aider à comprendre cette autre dimension de la violence actuellement perpétrée par la mara dans ses territoires. Harrison critique l'hypothèse selon laquelle les formes de "réciprocité négatives » (y compris la prédation physique que représente la violence et la prédation économique que représente l'exploitation) seraient occasionnées par la distance sociale, à savoir ce manque de liens entre les groupes et les individus. Selon lui, la violence ne témoigne pas forcément d'une séparation sociale, mais en revanche peut très bien en être productrice. Les mondes sociaux mélanésiens qu'il décrit dans ses travaux sont imprégnés d' "incontournables relations d'obligation » qui font que les groupes « cherchent sans cesse à s'extirper l'un de l'autre 53 ». Le problème pour ces groupes n'est donc pas d'établir des liens mais d'assurer des frontières. La violence leur donne ainsi un moyen parmi d'autres de délimiter et, le cas échéant, de couper ces liens d'obligation. L'usage de la violence permet de distinguer ceux qui vivent à l'intérieur de la sphère de réciprocité (positive) de ceux qui vivent à l'extérieur.

De la même manière, la mara peut elle aussi être considérée comme une sorte de groupe « intrinsèquement perméable 54 » aux prises avec « les liens

50. Cruz J.M., "Central American maras: from youth street gangs to transnational protection rackets ", Global Crime, vol. 11, n², 2010, p. 396.

51. Levenson D., op. cit., p. 3.

52. Cover R., « Nomos and narrative ", Harvard Law Review, vol. 97, n4, 1983, p. 31.

53. Harrison S., The Mask of War: Violence, Ritual, and the Self in Melanesia, Manchester, University of Manchester Press, 1993, pp. 23, 9. 
entrecroisés » qui existent entre des groupes en conflit et qui risquent de produire des « appartenances incompatibles » s'ils ne sont pas suspendus ou rompus ${ }^{55}$. Malgré la séparation physique provoquée par l'incarcération de bon nombre de membres, la distance sociale reste pour les cliques une chose inconfortable, fragile et artificielle. À l'intérieur des populations qu'ils exploitent, les membres ont des parents, des frères, des sœurs, des enfants, des amants, des amis, des connaissances, des gens qui leur apportent de l'aide, tous impliqués dans leurs propres réseaux axés sur l’appartenance et la réciprocité. Dans un paysage où les liens sociaux se multiplient et s'enchevêtrent, les actes de réciprocité négatives "seraient moins une conséquence de la distance sociale qu'un moyen d'en créer ou bien de faire appliquer cette distance 56 ». En tirant profit de sa propre communauté de façon prédatrice, la clique revendique et joue cette séparation, ce manque d'obligation.

Pour être capables de se livrer aux déprédations physiques et économiques qui leur sont demandées, les membres doivent en arriver à considérer les habitants de leur propre quartier comme des inconnus (au moins d'un point de vue éthique) auxquels ils ne doivent rien mais auxquels ils peuvent tout prendre. C'est dans ce but qu'ils ont recours à une violence transgressive qui leur permet de (re)créer les frontières de la réciprocité et de l'intimité. D'autant plus quand il s'agit des violences les plus extrêmes perpétrées par les gangs (le meurtre ou la mutilation de jeunes femmes dans le barrio par exemple) qui offrent ainsi un moyen de construire et de communiquer le rejet de tout sentiment de responsabilité envers le quartier et ses habitants. Arturo a décrit les rites et les critères d'initiation (qui, selon les dires de certains membres, peuvent inclure le viol collectif de jeunes filles ou le meurtre de victimes taboues, y compris des enfants ou des femmes enceintes) comme s'inscrivant dans « un processus visant à faire perdre leur âme (desalmar) aux membres ». Des anciens membres m'ont dit qu'à leurs yeux, ce processus serait conçu afin de pousser les recrues à violer les normes et les liens sociaux les plus fondamentaux pour restreindre tout sentiment d'appartenance, de responsabilité ainsi que tout réseau de soutien au gang. La violence perpétrée par la mara à l'encontre des habitants d'El Romero redéfinit ainsi la forme et la teneur des imaginaires sociaux locaux d'une manière qui se révèle aussi productive pour les gangs que destructrice pour le quartier.

54. Harrison S., op. cit., p. 14.

55. Roberts S., Order and Dispute: An Introduction to Legal Antbropology, New York, St Martin's, 1979, p. 56.

56. Harrison S., op. cit., p. 16. 
Autrefois, les membres des maras de Guatemala-ville semblaient considérer leur gang « tout simplement comme l'extension du quartier 57 ». Il représentait leur «barrio ». Toutefois, l'imaginaire social qu'ils partageaient naguère avec les autres habitants du quartier (cet accord fondamental qui trace les frontières de la légitimité et du tolérable, caractéristique du banditisme social et des économies morales) s'est étiolé dans le sillage du virage prédateur entamé par la mara vers une extorsion systématique. Au fur et à mesure que la mara se retire du monde social et éthique du quartier, le barrio des gangs se détache du barrio des communautés. Cette dérive normative a commencé quand les franchises transnationales ont pris le pas sur les quartiers locaux, transformant les coordonnées identitaires des gangs. Le choix fait par l'État guatémaltèque de préconiser l'incarcération à la place d'autres stratégies de répression a également contribué de façon déterminante à désencastrer les cliques et les membres des quartiers et à transformer les maras de Guatemalaville en agents impitoyables d'un entrepreneuriat de la violence.

L'extension de l'extorsion et l'élargissement simultané du répertoire d'actes violents de la mara ont modifié l'expérience de l'insécurité à l'intérieur des communautés qui vivent sous le contrôle de ces gangs, transformant la vie économique et sociale de façon radicale. En essayant d'expliquer ce qui leur est arrivé et les changements qu'ils constatent dans leur quartier ou dans les gangs qui les entourent, les habitants d'El Romero évoquent souvent la transformation du rapport gang-barrio ainsi que l'affaiblissement ou l'abandon des codes d'autrefois. L'histoire du déclin des códigos de barrio nous livre une histoire morale d'El Romero. Cette histoire raconte l'évolution de l'éthique de la violence de la mara et la transformation de bandits sociaux en professionnels du crime. Elle raconte en plus la détresse provoquée par une telle déstabilisation de la forme et des limites du monde normatif. En échangeant le « code du barrio » contre « l'idéologie d'une entreprise », et en tirant profit de façon prédatrice des mêmes quartiers auxquelles elle s'identifiait par le passé, la mara a transformé le paysage moral de la violence dans les zones rouges de la capitale. 